\title{
Les interactions entre parents et enseignants à l'entrée et à la sortie des classes lors de la première année d'école: deux fois dix minutes quotidiennes d'ambiguïté
}

Ogay, T., \& Ballif, L. (2019). Les interactions entre parents et enseignants à l'entrée et à la sortie des classes lors dela première année d'école: deux fois dix minutes quotidiennes d'ambiguïté. In A. Sieber Egger, G. Unterweger, M. Jäger, M. Kuhn, \& J. Hangartner, (Éd.), Kindheit(en) in formalen, nonformalen und informellen Bildungskontexten. Ethnografische Beiträge aus der Schweiz. (pp. 175-194). Wiesbaden : Springer VS.

https://link.springer.com/chapter/10.1007\%2F978-3-658-23238-2 9

Prof. Dr. Tania Ogay (Université de Fribourg, Département des Sciences de l'éducation)

- Rue P.-A de Faucigny 2, CH-1700 Fribourg

- Tania.Ogay@unifr.ch

- Domaines de recherche: Communication interculturelle, relation école - familles, formation interculturelle

Prof. Loyse Ballif (Haute école pédagogique de Fribourg)

- Rue de Morat 36, $\mathrm{CH}-1700$ Fribourg

- ballifloy@edufr.ch

- Domaines de recherche: Relation famille-école, enseignement en classe enfantine

\section{Résumé}

Dans les premières années d'école, la relation école-familles se construit largement lors des interactions informelles quotidiennes entre parents et enseignants, quand les enfants arrivent et repartent de l'école. Une recherche ethnographique montre combien ce moment apparemment anodin de l'entre-deux est empli d'ambiguïté et représente un enjeu pour la collaboration entre l'école et les familles. Dans ces brefs moments d'interaction, les acteurs sont nombreux, parfois changeants selon les accompagnants des enfants et le taux d'emploi des enseignants. Leurs rôles et motivations dans l'interaction sont divers, souvent contradictoires, jamais explicités et encore moins négociés. Chacun essaie de se mettre en scène comme un acteur compétent de la relation école-familles, un exercice particulièrement délicat pour les parents peu familiers de l'école.

\section{Mots-clés:}

Entrée à l'école, relation école-familles, groupes minoritaires, transition, ethnographie de l'éducation, communication interculturelle, interaction, temporalité

\section{Introduction}


L'entrée à l'école est une étape marquante dans la vie d'un enfant, comme de ses parents. C'est le début de la relation école-familles, dont la qualité n'est pas sans importance pour le déroulement de la scolarité (Patrikakou et al. 2005). Le tout premier jour d'école consacre la transition qu'opère l'enfant soudainement devenu aussi élève ${ }^{1}$ - entre l'espace familial, privé, et l'espace de l'école, celui de la société; il constitue ainsi un rite de passage (van Gennep 1909) entre la période de la petite enfance et celle de l'enfance. Pour les parents dont c'est le premier enfant qui entre à l'école, c'est aussi le moment où se concrétise leur nouveau rôle de parents d'élève, qu'ils seront appelés à jouer pendant de nombreuses années. Ainsi, lorsque l'enfant entre à l'école, c'est aussi l'école qui entre dans la vie des familles, bouleversant l'équilibre de la niche développementale (Super et Harkness 1986) autour de l'enfant. Les parents doivent alors apprendre à composer avec l'entrée en jeu d'un nouvel acteur de l'éducation de leur enfant, et non des moindres.

La transition que représente l'entrée à l'école entraîne l'enfant et aussi ses parents dans une dynamique de changement et de développement: comme le formule Zittoun (2012) pour les nombreuses transitions qui interviennent au cours d'une vie, trois types de processus sont alors engagés: d'apprentissage (développement de nouvelles manières de comprendre et d'agir), de changement identitaire (liés à des enjeux de reconnaissance et d'estime de soi) et de construction de sens (permettant d'inscrire cette rupture dans le cours de sa vie). Si l'enfant qui entre à l'école est bien entendu au cœur de la transition qui l'amène à passer - physiquement et psychologiquement - quasi quotidiennement d'un monde à un autre, le modèle écologique et dynamique de Rimm-Kaufman et Pianta (2000) considère que la transition implique également les autres acteurs du système écologique (Bronfenbrenner 1986) dans lequel est inséré l'enfant: sa famille, l'école, mais aussi les pairs et le voisinage. La recherche dont nous rendons compte ici (Ogay 2017a) ${ }^{2}$ 'sest focalisée sur les parents et les enseignants, cherchant à comprendre comment se construit leur relation qui donne pour ainsi dire forme au contexte dans lequel l'enfant/élève réalise sa transition entre la famille et l'école. En effet, comme le relèvent Rimm-Kaufman et Pianta, au fil du temps et des interactions, des "patterns" (2000, p. 499) de relations se développent entre les différents acteurs de la transition, facilitant ou au contraire compliquant l'adaptation de l'enfant à l'école et, au final, sa réussite scolaire. A l'heure où les discours scientifiques comme institutionnels appellent à la collaboration et même au partenariat entre l'école et les familles, considérés comme favorables à la réussite scolaire (voir par exemple Hampden-Thompson et Galindo 2017), comment parents et enseignants parviennent-ils à s'accorder pour soutenir l'enfant dans sa scolarité ? Et ceci en particulier quand l'asymétrie presque inévitable de leurs statuts - les enseignants étant des professionnels à qui la société confie la mission de former les citoyens de demain - se trouve encore accrue par le manque de références culturelles communes lorsque les enseignants sont issus du groupe social majoritaire et les parents de populations immigrées et/ou aux revenus modestes?

La perspective théorique générale que nous adoptons pour étudier la relation entre l'école et les familles est celle de la communication interculturelle, en particulier le cadre d'analyse sémiopragmatique de la communication développé par Frame (2013) qui allie la communication interculturelle (notamment la

\footnotetext{
${ }^{1}$ Que nous désignerons par le syntagme enfant/élève, symbolisant cette double appartenance.

${ }^{2}$ Recherche financée par le Fonds national suisse de la recherche scientifique, n de projet 100019_152695.
} 
Théorie de l'accommodation de la communication de Gallois, Ogay, et Giles 2005) et l'interactionnisme symbolique (Mead 1934; Blumer 1969). Nous nous intéressons ainsi au processus de la communication dans les interactions entre parents et enseignants, où ces acteurs échangent et co-construisent du sens (le processus de 'meaning-making' central dans l'interactionnisme symbolique) et mettent en scène leurs identités (Goffman 1973) à partir de leurs interprétations de la situation ainsi que de leurs propres cadres de référence a priori, cette co-construction de sens étant préfigurée - et donc contrainte - par le contexte culturel dans lequel est insérée la relation. Précisons encore que dans la conception pragmatique de la communication, qui étudie comment la communication affecte le comportement, il n'y a pas nécessairement un échange verbal. En effet, tout est communication : comme le formule le premier des cinq axiomes de la communication humaine selon Watzlawick, Beavin et Jackson (1972), on ne peut pas ne pas communiquer. Ainsi, même des parents et des enseignants qui n'échangent aucune parole, ni même un regard voire qui se tiennent à distance, sont en train de communiquer et de construire leur relation.

Dans ce chapitre, nous analysons en particulier la communication entre parents et enseignants lors des moments d'arrivée et de départ de l'école, observés tout au long de la première année d'école. En effet, une fois passé le jour de la 'rentrée', une fois éteints les flashs des appareils photos et retombées les émotions exacerbées par la conscience de l'importance de ce moment particulier dans la vie de tout enfant et de tout parent, la transition se rejoue quotidiennement tout au long de la première année scolaire (Rimm-Kaufman et Pianta 2000). Chaque jour, lorsque le jeune enfant/élève arrive à l'école et en repart, accompagné de ses parents ou d'autres adultes, d'un grand frère ou d'une grande sœur, il passe d'un monde à un autre. Chaque jour, lors de ces moments apparemment anodins d'arrivée et de départ où se transmet le témoin de la responsabilité de la sécurité et du bien-être de l'enfant/élève, parents et enseignants tissent leur relation au gré de leurs interactions (ou de leurs non-interactions) et construisent ainsi la collaboration entre l'école et les familles. D'une durée d'à peine plus de 10 minutes à chaque fois, ces moments font partie des 'temps interstitiels' de l'école dont Marcel (2002) a souligné la richesse pour l'étude des dynamiques relationnelles entre les différents acteurs scolaires. Lors de la conception de notre recherche, nous n'avions tout d'abord vu dans ces temps d'arrivée et de départ de l'école qu'une occasion pour observer des interactions informelles entre les parents et les enseignants. Ce n'est que progressivement, au gré des observations et de leur analyse, que nous est apparue la signification particulière que prennent ces deux fois dix minutes de transition et que nous avons pris conscience de l'enjeu qu'elles représentent pour l'instauration d'une collaboration entre l'école et les familles.

Comme pour le tout premier moment de rencontre entre les familles et les enseignantes, deux mois avant le début de l'école lors d'une visite de la classe et d'un atelier censés préparer l'entrée à l'école (Ogay 2017b), les moments d'arrivée et de départ de l'école pendant cette première année scolaire nous sont apparus comme caractérisés par une forte ambiguïté, pour les enfants comme pour leurs parents. II n'y a en soi rien d'étonnant à cela : la transition, le passage d'un espace, d'un temps à l'autre, est par essence un moment d'ambiguïté, où les identités comme l'interprétation du sens à donner à la situation et aux comportements des uns et des autres sont incertaines: que faudrait-il faire/dire, et comprendre? et comment? Pour l'analyse, la question qui se pose est ce qu'il advient de l'ambiguïté inhérente à cette 
situation de transition: que font les acteurs pour la gérer, la contenir, la transformer? Selon Gudykunst et sa Théorie de la gestion de l'incertitude et de l'anxiété (2005), une situation d'interaction ambiguë produit de l'incertitude et de l'anxiété qui, lorsqu'elles sont excessives, peuvent mettre à mal la qualité de la communication et donc son efficacité: le risque est alors grand que les acteurs ne se comprennent pas, fassent des attributions (Deschamps et Beauvois 1996) erronées et ne retirent que frustrations de leurs échanges. Ces temps interstitiels où se passe la transition sont également marqués par des enjeux identitaires et de gestion de la face (Ting-Toomey 2004) importants: dans cette rapide scène quotidienne, chacun, enfants comme adultes, tente en effet de faire bonne figure et de se mettre en scène (Goffman 1973) comme un acteur compétent de la relation école-familles. Si les enseignants sont dans un environnement qui leur est familier, dont ils connaissent les codes et sont les chefs d'orchestre, il n'en va pas de même des parents. À fortiori lorsque ceux-ci n'ont pas eux-mêmes connu cet établissement ou ce système éducatif, ou lorsqu'ils sont peu à l'aise avec le monde scolaire et peu familiers de ses normes et implicites: l'entrée à l'école de leur premier enfant les place devant le défi de réussir leur entrée en scène, sans posséder le script de la pièce qui se joue. Comme l'observe Périer en France avec des familles "aux marges de l'école" (2017, p. 229), certains parents ne franchiront même jamais le seuil de l'école:

Cet effet de seuil symbolise le basculement d'un statut de parent à celui de parent d'élève, et la possibilité ou le risque d'être interpellé à ce titre. L'école se présente alors comme un espace, aussi accueillant soit-il, de mise en jeu de soi, de son image et de son identité. Les parents les plus vulnérables vivent ce rapport sur le mode d'une confrontation inégale où ils redoutent que le dévoilement de leur incompétence ne les disqualifie. Les moins 'habilités' d'entre eux, c'est-à-dire les moins assurés de leur valeur dans le lieu qu'ils fréquentent, se montrent volontiers hésitants et inclinent à se soustraire au regard d'autrui, au risque que cette absence ou vacance ne vienne renforcer les préjugés à leur encontre. (Périer 2015, p. 11)

La relation entre parents et enseignants est ainsi grevée d'une asymétrie à sa base, augmentée du fait que tout se joue très rapidement lors des moments interstitiels: non seulement leur durée est très courte, mais les parents se trouvent en concurrence entre eux pour entrer en relation avec l'enseignant. Il y a donc une sorte de double handicap de départ pour les parents: se trouver démunis mais exposés à un jugement, et devoir faire vite. Tandis que les enseignants, certes aussi exposés, ont plus de pouvoir sur le choix des codes et des temporalités.

\section{La construction de la relation entre l'école et les familles sous la loupe d'une recherche ethnographique}

Chercher à percer le processus de construction d'une relation sociale entre des acteurs insérés dans un contexte culturel forcément complexe demande un outillage théorique et méthodologique qui permette une granularité fine. En abordant la relation école-familles dans une perspective de communication interculturelle, nous cherchons à faire émerger la co-construction de sens par les acteurs de la relation école-familles au cours de leurs interactions. Nous cherchons ainsi à saisir comment, à partir notamment de leurs propres références culturelles et personnelles, ils s'adaptent continuellement les uns aux autres et à ce que chacun comprend de leur relation en train de se construire. Au niveau méthodologique, cette granularité fine est apportée par la démarche ethnographique (voir notamment Payet 2016) que nous avons adoptée pour observer la construction de la relation école-familles dans un établissement scolaire francophone du canton de Fribourg (Suisse). Un critère important de sélection de cet établissement est 
qu'il accueille une population majoritairement issue de la migration, aux revenus modestes et avec d'autres langues que le français comme langue(s) familiale(s). Pendant 3 années scolaires (de 2012 à 2015), nous avons observé la construction de la relation entre les 6 enseignantes des 4 classes accueillant les élèves de $1^{\mathrm{e}}$ et de $2^{\mathrm{e}}$ année de scolarité et les parents de 22 élèves qui entraient alors à l'école (à l'âge de 4 ans). Les outils de récolte des données étaient :

a) l'observation participante des moments d'interactions formels (visite de classe et atelier de préparation à l'entrée à l'école, réunions de parents, classes ouvertes, entretien enseignantesparents lors du premier bulletin semestriel) et informels (les moments de transition à l'arrivée et au départ de l'école, quelques sorties scolaires),

b) l'entretien de recherche semi-directif avec les parents (à domicile) ainsi qu'avec les enseignantes (en classe),

c) la collecte des documents remis aux parents par les enseignantes.

Au total, 195 rapports d'observation ont été produits et 101 entretiens de recherche (68 auprès des parents, 30 auprès des enseignantes et 3 auprès d'autres acteurs) ont été réalisés et intégralement transcrits. L'analyse des données a été réalisée à l'aide du logiciel NVivo, dans une démarche inspirée par Miles et Huberman (2003) ainsi que Paillé et Mucchielli (2012).

\section{L'entrée et la sortie des classes : les prescriptions et leurs interprétations dans l'établissement observé}

La période à laquelle a été réalisée la recherche correspond à celle où le canton de Fribourg révisait sa loi scolaire. Formellement, les données ont été récoltées sous l'ère de la loi de 1985 (État de Fribourg 1985) et de son règlement d'exécution (État de Fribourg 1986). Cependant, la loi de 2014 (État de Fribourg 2014), entrée en vigueur en août 2015 quand nous terminions notre récolte de données, ainsi que son règlement d'exécution (État de Fribourg 2016) sont bien présents dans le contexte scolaire et sociétal au moment de nos observations, même si seulement officieusement. Si la collaboration entre les parents et l'école est déjà prônée dans la loi de 1985 (article 31), la nouvelle loi la renforce en affirmant une responsabilité conjointe des tâches d'éducation et d'instruction de l'enfant (article 2 alinéa 1, article 30 alinéa 1) ainsi qu'en prévoyant la création de conseils des parents pour chaque établissement (article 31). Quant au règlement accompagnant la nouvelle loi, il mentionne les modalités d'information des parents par l'école (entretiens individuels, bulletin scolaire, séances d'informations et communications écrites) mais sans en préciser la fréquence ni même le caractère obligatoire ou facultatif. Si les premiers alinéas des deux textes de loi comme du règlement de 2016 se montrent ambitieux car semblant aspirer à une collaboration de type partenarial entre l'école et les familles, les alinéas suivants indiquent cependant que le législateur estime que l'école et les familles peuvent se seconder l'un l'autre dans l'éducation de l'enfant/élève par la seule information réciproque, ce qui correspond au premier des quatre niveaux de collaboration identifiées par Larivée, Kalubi et Terrisse (2006). Ainsi, malgré des ambitions affichées allant dans le sens d'un partenariat entre l'école et les familles, nous avons pu observer que les enseignants fribourgeois - au moment de notre recherche du moins - avaient finalement très peu d'indications sur ce que leur employeur attend d'eux pour susciter et entretenir cette collaboration avec les parents d'élèves. Seul l'entretien de fin de premier semestre (analysé dans Nunez Moscoso et Ogay 2016; Scalambrin et Ogay 2014; Conus et Ogay soumis et révisé) est explicitement prescrit et scrupuleusement réalisé par les enseignantes de l'établissement observé. 
Pour ce qui est du temps d'entrée et de sortie des classes, s'il fait bien l'objet d'une prescription dans le nouveau règlement, ce n'est pas pour son potentiel de création et de soutien du lien entre l'école et les familles mais pour la question juridique de la responsabilité de l'enfant/élève dans cet espace-temps d'entre-deux. Ainsi, le règlement de la loi scolaire précise dans son article 32 consacré au temps scolaire: «Durant le temps scolaire, ainsi que dix minutes avant et après les cours, les élèves sont placés sous la responsabilité et la surveillance de l'école» (alinéa 2). Quant à l'article 122, il lie le territoire de l'école avec le temps de la transition: «Les communes définissent, en collaboration avec la direction d'établissement, le périmètre de chaque établissement dans le règlement scolaire communal. Ce périmètre délimite l'aire dans laquelle les élèves sont placés sous la responsabilité de l'école durant le temps scolaire.» (État de Fribourg 2016). Notons que même si ce règlement n'était pas encore en vigueur au moment de notre recherche, les enseignantes nous parlent expressément de leur responsabilité de surveillance pendant ce temps de transition avant et après la classe. Par contre, les parents à qui nous avons posé la question n'étaient pas au courant de cette règle des 10 minutes, ni non plus de celle leur interdisant l'accès à l'intérieur du bâtiment scolaire. Ils ont cependant reçu cette information le jour de la rentrée, dans un document émanant des autorités scolaires communales, parmi beaucoup d'autres documents (tous en français):

\section{Discipline à l'intérieur et à l'extérieur des bâtiments}

Afin de respecter la tranquillité et le bon déroulement des leçons, les parents, et tout particulièrement ceux des élèves des classes enfantines, qui viennent chercher leurs enfants à la sortie de l'école sont priés de les attendre devant le bâtiment scolaire et non dans les couloirs jouxtant les salles de classe.

En outre, les automobilistes sont tenus de respecter les dispositions sur le parcage et de ne pas empiéter sur les emplacements réservés à la récréation ou au passage. Nous rappelons que les enseignants surveillent les abords des écoles 10 minutes avant et après l'école. Veuillez ne pas envoyer vos enfants trop tôt à l'école.

(Recommandations et directives aux parents 2012-2013)

Dans l'établissement observé, les élèves en première année de scolarité vont à l'école tous les jours ouvrables de la semaine, soit 4 après-midis (durée de la classe: 2h10) et 1 matinée (3h15). Pour 3 de ces demi-journées, notons que les élèves de $1^{\mathrm{e}}$ année sont en classe ensemble avec ceux de $2^{\mathrm{e}}$ année, ce qui double le nombre d'enfants/élèves et d'accompagnants présents lors de ces 10 minutes (sur les 3 années observées, l'effectif moyen des classes observées est de 17 élèves, avec une proportion égale d'élèves en $1^{\mathrm{e}}$ et $2^{\mathrm{e}}$ année de scolarité).

Deux modes d'organisation différents de ces moments de transition ont été observés, liés notamment aux spécificités architecturales de l'espace construit dans lequel se trouvent les classes:

- 2 classes occupent seules un petit pavillon situé aux confins de l'établissement. Un escalier d'une dizaine de marches permet d'accéder au vestiaire commun depuis un étroit chemin, bordé de buissons épineux empêchant l'accès direct à un parking public. L'espace est restreint, plusieurs parents ${ }^{3}$ se tiennent à distance d'un côté ou de l'autre de l'escalier, notamment lorsqu'ils sont avec des enfants plus jeunes, souvent en poussette. L'exiguïté des lieux rend impossible la confidentialité des échanges entre les enseignantes et les parents proches de l'escalier. D'autant plus que les 3 enseignantes accueillent et surtout libèrent leurs élèves en se tenant en haut de l'escalier ou à mi-hauteur de la rampe: ceci leur permet de conserver une vue générale de la

\footnotetext{
${ }^{3}$ Ou d'autres accompagnants, précision qui ne sera en principe plus apportée dans la suite du texte.
} 
scène et de surveiller ainsi les départs de leurs élèves, mais gêne les interactions avec les parents. Lors de l'accueil, pendant les premiers jours d'école, elles descendent au bas de l'escalier et forment la 'colonne par deux', chaque classe de son côté de l'escalier, ce qui a pour effet de repousser les parents contre la haie ou plus loin encore sur le chemin. A la sortie de classe, les enseignantes - qui revêtent, au début de l'année scolaire surtout, le gilet de couleur fluorescente symbolisant les 10 minutes de surveillance ${ }^{4}$ - saluent leurs élèves (par une poignée de main et un regard "dans les yeux» souvent difficiles à obtenir de ces tout jeunes enfants) enhaut de l'escalier lorsqu'ils sortent du vestiaire. En principe, elles ne laissent les élèves descendre l'escalier que si elles ont vu la personne venue les chercher. Cependant, au vu du nombre élevé d'élèves qui sortent en même temps du vestiaire ainsi que de l'espace et du temps limités, ces deux étapes de la sortie se révèlent difficiles à respecter et leur application au quotidien est souvent approximative. D'autant plus que le nombre d'élèves et d'accompagnants double une fois les premiers jours d'école passés : en effet, les enseignantes du pavillon mettent alors en place une surveillance alternée des élèves de leurs deux classes au début comme à la fin de l'école, ce qui signifie qu'en plus de leurs élèves, elles sont censées veiller également sur les allées et venues des élèves de l'autre classe.

- Les 2 autres classes accueillant des élèves dans leur première année de scolarité sont situées dans le bâtiment principal de l'établissement, à deux étages différents. Les moments d'accueil et de sortie se passent dans la cour de l'école, au milieu de l'animation créée par les élèves de tous les degrés de la scolarité obligatoire qui fréquentent le même bâtiment. La surveillance des élèves prescrite par le règlement est assurée en tournus par tous les enseignants du bâtiment principal, qui portent alors le gilet orange ad hoc. A l'abri d'un auvent, les 3 enseignantes des 2 classes de $1^{\mathrm{e}}$ et $2^{\mathrm{e}}$ années ont apposé des affiches qui identifient un espace de rencontre pour chacune de leurs classes. Les enseignantes viennent y chercher leurs élèves, les parents forment souvent un cercle autour des enfants qui se regroupent peu à peu, aidés par les enseignantes et parfois aussi par les parents, pour former la 'colonne par deux' qui se dirigera ensuite vers l'une des deux portes du bâtiment. A la sortie, les enseignantes accompagnent leurs élèves dans la cour. Malgré le nombre important de personnes présentes dans la cour et l'animation qui y règne pendant ces 10 minutes, l'espace est généreux, laissant à chacun la possibilité de se placer selon son envie. Ainsi, l'espace disponible permet aux enseignantes et aux parents d'avoir des échanges qui peuvent rester relativement confidentiels tout en laissant la possibilité aux enseignantes de garder un œil sur les départs de leurs élèves, même si elles ne voient pas toujours tout. Au cours du $2^{\mathrm{e}}$ semestre, certaines enseignantes laissent parfois leurs élèves descendre seuls dans la cour après la classe, valorisant l'autonomie des élèves mais restreignant ainsi les possibilités d'interaction avec les parents. Elles ne contreviennent alors pas à la règle des 10 minutes car il y a toujours un enseignant qui surveille la cour, mais celui-ci ne contrôle pas avec qui partent les élèves.

\footnotetext{
${ }^{4}$ Auquel fait écho le sautoir orange (appelé 'triangle') que reçoivent les enfants/élèves pour le trajet entre le domicile et l'école, une mesure de sécurité routière recommandée par l'école aux parents, qui l'appliquent de façon plus ou moins rigoureuse.
} 
- Notons que quelques parents, dans les deux bâtiments, cessent parfois d'accompagne leur enfant qu'ils laissent aller et revenir seul de l'école, en particulier lorsqu'ils habitent tout près et peuvent surveiller le trajet de leur enfant depuis le balcon ou une fenêtre de leur domicile.

Nous pouvons ainsi remarquer que les aménagements physiques de l'espace-construit contraignent la proximité ou distance entre les enseignantes et les parents; selon les termes du cadre d'analyse sémiopragmatique de la communication de Frame (2013), ils configurent leurs interactions. Mais nous constatons également que les acteurs élaborent des stratégies pour rétablir la 'bonne distance' lorsqu'elle est menacée par l'espace-construit: se tenir éloigné (en hauteur sur l'escalier, à distance sur le chemin ou dans la cour d'école), ou au contraire se rapprocher (afficher un lieu de rencontre dans la cour de l'école, descendre ou monter l'escalier). Certains objets balisent ainsi ces espaces, favorisant les rapprochements ou au contraire maintenant la distance: la haie, l'escalier, la porte du bâtiment. À l'exemple du gilet de couleur porté par les enseignants, ces objets sont également des marqueurs temporels puisque leurs accès et usages sont organisés selon un horaire précis : hors des 10 minutes de transition, les enseignants ne portent pas le gilet, les portes du bâtiment se ferment (dans le grand bâtiment, elles sont verrouillées quelque temps après la sortie des classes). La transition quotidienne entre familles et école se déroule ainsi dans un espace-temps spécifique qui, pour reprendre les termes de l'architecte et urbaniste Soulier (2012), délimite (la 'frontière') ou relie (le 'frontage') espace privé et espace public. Parlant d' "espaces de bordure, d'interface, de transition», les géographes Da Cunha et Guinand $(2014,50)$ relèvent l'importance sociale des espaces de frontage qui permettent le lien: "A la limite de la ligne, opaque ou ouverte, qui sépare l'espace privé du domaine public, ils constituent une composante importante du système de déplacements mais aussi du tissu social». On peut remarquer que cette ligne qui délimite le périmètre dans lequel les élèves sont sous la responsabilité de l'école est, pour les classes du grand bâtiment, relativement claire (signalée par une barrière et un portail d'un côté de la cour, mais sans limite claire de l'autre côté) et éloignée du lieu de rencontre, celle-ci se passant clairement sur le territoire de l'école. Par contre, pour le petit bâtiment, cette ligne est floue: l'étroit chemin sur lequel se tiennent les parents est également un lieu de passage public, la transition se passe ainsi directement sur la ligne de frontière.

\section{4 'Relationner' ou contrôler?}

Nos analyses nous ont permis d'identifier une tension qui transparaît dans les discours et comportements des acteurs de ces moments d'accueil et de sortie des classes: la tension entre le souhait de mettre à profit ces moments de coprésence entre parents et enseignantes pour construire leur relation (objectif que nous formulons de façon brève en proposant 'relationner'), et la mission de surveillance des enfants/élèves (objectif 'contrôler'). Cette tension s'observe chez les enseignantes comme chez les parents.

\subsection{Relationner - contrôler, du côté des enseignantes :}

Les enseignantes nous disent à plusieurs reprises dans nos entretiens valoriser les moments interstitiels pour les contacts informels qu'elles peuvent y avoir avec les parents et ainsi créer le lien nécessaire à la collaboration école-familles. Une enseignante se montre particulièrement à l'aise dans ces contacts informels dont elle prend souvent l'initiative, également avec les parents d'élèves (actuels comme passés) parlant peu le français. Mais nous avons observé que ces moments privilégiés se passent 
principalement lorsque les enseignantes sont dans la cour au-delà des 10 minutes de surveillance, par exemple quand elles restent avec un élève dont les parents sont en retard. Pendant les 10 minutes de l'accueil et du départ de l'école, nous les voyons surtout accaparées par des objectifs de contrôle :

- Du comportement des élèves: équipement correct (habits, matériel scolaire), formation de la 'colonne par deux', rite de salutation, pas de jeux dangereux (sur l'escalier pour le petit bâtiment, au toboggan pour le grand), pas de bagarres ni de bruit excessif.

- Du comportement des accompagnants: bien sûr nettement moins fréquents que le contrôle des élèves, nous avons observé quelques contrôles des accompagnants par les enseignantes, au sujet notamment du matériel ou du respect d'une consigne (une enseignante empêche par exemple une maman de prendre des photos le jour de la rentrée, invoquant un règlement sur le droit à l'image des autres personnes présentes). Le contrôle des accompagnants par les enseignantes est plus explicite lorsqu'il s'agit des grands frères ou grandes sœurs qui sont souvent réprimandés lorsqu'ils arrivent en retard, même quand ces retards sont dus au fait que leurs propres enseignants les ont libérés après l'heure de fin des classes. Nous n'avons par contre jamais observé de remarques faites par les enseignantes aux quelques parents qui contreviennent à la règle leur interdisant l'accès au bâtiment scolaire.

- Du départ des enfants/élèves avec les bons accompagnants. Selon nos observations, cet objectif de contrôle prend une place très importante, il configure fortement le comportement des enseignantes pendant les moments interstitiels de sortie de classe. Ce contrôle répond à leur interprétation du règlement scolaire au sujet de la surveillance des dix minutes avant et après la classe. Cette interprétation, qui répond également à l'attente de plusieurs parents, correspondelle à l'intention des auteurs du règlement scolaire? Est-elle réaliste? Une interprétation plus minimaliste que celle qu'en font les enseignantes pourrait être que l'école est responsable de la sécurité des élèves dans le périmètre scolaire pendant ces dix minutes, les enseignants devant ainsi veiller à ce que les élèves ne se blessent pas (notamment sur le toboggan ou sur l'escalier, causes de nombreuses interventions des enseignantes), ou qu'il n'y ait pas de bagarres. Mais estil de leur responsabilité de s'assurer de la 'remise' correcte des enfants/élèves? II s'agit d'une tâche qui n'a rien d'anodin: pour être en mesure de l'assurer, les enseignantes doivent bien sûr non seulement connaître les élèves mais aussi quels sont leurs parents et/ou accompagnants, or il n'est pas rare qu'il y ait des changements. Une des enseignantes du petit bâtiment demande ainsi aux parents de sa classe de remplir un formulaire en début d'année pour indiquer qui vient chercher l'enfant/élève quel jour, et leur demande de la tenir informée des changements (durables comme momentanés). Elle résume cette information sur une petite fiche qu'elle consulte lors des moments de sortie de classe. Cependant, ni elle ni ses collègues de l'autre classe ne se transmettent ces informations lorsqu'elles mettent en œuvre leur système de surveillance alternée qui implique pourtant qu'elles ont à contrôler les départs des élèves des deux classes en même temps.

\subsection{Relationner - contrôler, du côté des parents}

Avant le début de l'école, les parents nous ont confié lors des entretiens semi-structurés réalisés à leur domicile s'attendre à pouvoir parler avec l'enseignante lors des moments de transition, un peu comme plusieurs en ont eu l'habitude pour la crèche ou pour la structure d'accueil extrafamilial des enfants de 3 
ans créée par la commune pour les familles du quartier. Ils espèrent ainsi avoir un écho du comportement et des apprentissages de leur enfant en classe, mais aussi créer un lien avec l'enseignante avec qui ils souhaitent collaborer. Une fois l'année scolaire commencée, les parents font cependant rapidement le constat que le contexte des temps interstitiels (beaucoup de gens, peu de temps, et un espace trop exigu pour un des bâtiments) est peu favorable aux échanges informels, et que seuls les échanges 'utiles' - pour communiquer une information par exemple - sont possibles, comme le décrit cette mère:

Pas difficile, mais... ce n'est pas qu'on peut parler beaucoup, parce qu'elles sortent juste avant que les enfants soient dedans. Et pis si on, si on doit dire quelque chose, on doit dire vite, parce que les enfants ils attendent. On n'a pas un moment. (entretien famille A, 01.07.2013. Emphase par la répondante).

La plupart des parents renoncent alors à leur objectif de relationner et ne cherchent pas à initier le contact avec l'enseignante, voire restent en retrait de la scène où se joue la transition. Ils se montrent soucieux de ne pas compliquer encore plus la tâche des enseignantes dont ils voient bien la complexité. Ils cherchent d'autant moins le contact qu'ils ont entendu les enseignantes énoncer à plusieurs reprises le principe 'pas de nouvelles, bonnes nouvelles' qui relie l'interaction entre parents et enseignantes à l'existence de problèmes (comme analysé spécifiquement par Conus et Fahrni 2017 dans le cadre de la même recherche). N'oublions pas non plus que les parents ont aussi dans ces moments de transition leurs propres préoccupations liées à l'objectif de contrôle, qui rend difficile la recherche de contact avec l'enseignante:

- De leur enfant/élève: contrôle de son comportement (qu'il maîtrise le rite des salutations de l'enseignante, qu'il tienne la main du camarade désigné par l'enseignante dans la 'colonne par deux', qu'il ne perturbe pas); contrôle également de son matériel (qu'il ait les bons habits et les ait enfilés correctement, qu'il ait le sac de gymnastique, le sac de la bibliothèque, le 'triangle', etc.). Derrière le contrôle de leur enfant se joue pour les parents l'enjeu de leur image de bons parents, aux yeux de l'enseignante comme aux yeux des autres parents. Nous assistons ainsi à plusieurs scènes où des parents interagissent avec leur enfant - souvent dans une langue autre que le français - alors qu'il est déjà ou encore sous le contrôle de l'enseignante (pour lui dire de regarder l'enseignante au moment de la saluer, de tenir la main de leur camarade dans la file, d'écouter la maîtresse quand elle leur donne des consignes avant de les libérer, etc.).

- Des éventuels frères et sœurs de l'enfant/élève: quand ceux-ci sont plus petits, les parents doivent également y porter leur attention et s'inquiètent aussi qu'ils ne perturbent pas (par exemple en montant l'escalier pour ceux qui ne sont pas dans une poussette). Les petits frères ou sœurs n'ont d'ailleurs pas forcément besoin d'être présents pour préoccuper leurs parents: une mère nous dit être tendue dans ces moments d'arrivée ou de départ de l'école quand le cadet dort seul à la maison (les horaires de l'école et de la structure d'accueil préscolaire du quartier n'étant pas coordonnés). Elle arrive alors à la dernière minute à l'école, voire parfois en retard, et repart au plus vite. Les parents ou les assistantes parentales ('mamans de jour') sont parfois aussi accompagnées par d'autres enfants/élèves, qu'il faut surveiller tout en confiant le premier à l'enseignante, puis conduire à l'autre bâtiment sans prendre de retard.

Lors des 10 minutes de transition, les acteurs semblent ainsi hésiter entre des missions et attentes contradictoires, ce qui résulte en des interactions parfois décalées, inadéquates, qui créent un malaise 
dans la relation. Le décalage est notamment rendu manifeste par l'utilisation de l'espace de l'interaction, qui devrait être proche pour l'objectif de relationner, et distant pour l'objectif de contrôle (par exemple une enseignante qui cherche à créer de la proximité avec un papa en lui lançant une remarque humoristique, mais à distance depuis le haut de l'escalier, sans la moindre intimité, et qui apparaît donc déplacée). Si le contrôle prend généralement le pas sur le relationnel (par exemple une enseignante qui parle à une maman tout en regardant ailleurs pour surveiller la scène de la remise des enfants/élèves), le relationnel - ou l'envie d'établir une relation - perturbe néanmoins la réalisation efficace du contrôle: il suffit de quelques secondes d'interaction avec un parent pour qu'un enfant/élève échappe à la surveillance de l'enseignante et s'en aille seul.

\section{Quand les impensés et les non-dits renforcent l'ambiguïté initiale de la transition}

Comme vu plus haut, toute transition est par essence un moment de fragilité, par l'ambiguïté des identités et des significations qu'elle implique. On retrouve cette idée dans le concept de 'liminarité' développé par Turner (1990; voir aussi Dartiguenave 2012) à partir de l'analyse des rites de passage par van Gennep (1909) : la transition se joue dans un entre-deux, un intervalle - ici les 10 minutes à l'entrée ou à la sortie des classes - où les identités et positions sociales sont indéfinies car flottantes entre deux statuts (notamment enfant/élève, mais aussi parent/parent d'élève, et même enseignant seul devant ses élèves et enseignant en présence de parents d'élèves), et donc ambiguës. Comment cette ambiguïté initiale des moments d'arrivée et de sortie de classe est-elle gérée par les enseignantes de notre terrain d'observation? Force nous est de constater que l'ambiguïté n'est pas reconnue, et donc encore moins gérée. Au contraire, le manque de conscience (de 'mindfulness' selon le terme de la théorie de William B. Gudykunst 2005) de la complexité de ce moment à l'apparence faussement anodine (quoi de plus simple en effet que d'apporter puis de reprendre son enfant à l'école, comme on le ferait d'un paquet à l'office postal?) vient renforcer encore l'ambiguïté déjà présente au départ et n'aide pas les parents à construire leurs repères.

Ainsi, la tension entre l'ambition maintes fois exprimée dans nos entretiens par les enseignantes de créer du lien avec les parents pendant les moments interstitiels et leur préoccupation du contrôle de la remise des enfants/élèves aux bons accompagnants renforce considérablement l'ambiguïté de ces 10 minutes de transition. Si quelques parents se disent satisfaits du contrôle des départs et se sentent en confiance, d'autres - en particulier du petit bâtiment - en repèrent vite les failles. Ils en sont d'autant plus insécurisés que certains éléments, comme le questionnaire leur demandant avec qui part l'enfant/élève ou aussi quelques sorties de classe où les enseignantes se montrent effectivement très strictes dans le contrôle des départs, semblent affirmer qu'il s'agit bien d'une tâche faisant partie de la mission des enseignantes. Les enseignantes ne semblent pas s'être accordées sur l'interprétation à donner de la prescription de surveillance des 10 minutes inscrite dans le règlement scolaire, ni sur ses implications pratiques (notamment sur la transmission des informations à l'enseignante qui assure la surveillance du départ des élèves). Leurs pratiques par rapport à cette mission ne sont pas identiques (entre elles, mais aussi pour une même enseignante d'un jour à l'autre), ce qui renforce l'ambiguïté pour les parents qui les observent. La signification de la mission de surveillance lors des 10 minutes n'a pas non plus été explicitée et encore moins négociée avec les parents: si l'information leur a été communiquée dans la documentation écrite reçue le jour de la rentrée, sa signification n'est pas explicitement traitée par les enseignantes (elle n'est qu'accidentellement et rapidement évoquée lors d'un des deux ateliers de 
préparation à l'entrée à l'école que nous avons observés, suite à une question de la 'coordinatrice écoleparents migrants' de la commune). Outre la question de la possibilité même et de l'organisation de la surveillance des départs des enfants/élèves, une autre ambiguïté est causée par la non-explicitation de ce que signifie la coprésence des enseignantes et des parents dans cet espace-temps de la transition: en particulier, qui est responsable du contrôle des comportements des enfants/élèves? Comment gérer ce double contrôle? Les parents sont-ils censés rester jusqu'à la fin des 10 minutes de l'accueil (mais quel est alors leur rôle?), ou partir dès que l'enseignante est présente? En l'état, les rôles respectifs des coéducateurs de l'enfant/élèves dans ces moments de transition sont confus et jamais négociés.

Malgré la charge que représente leur mission de surveillance pendant ces 10 minutes, surtout dans I'interprétation maximaliste qu'elles en ont, les enseignantes nous disent dans les entretiens avoir l'impression d'être à disposition des parents dans ces moments pour créer ce lien interpersonnel nécessaire à la collaboration. Cependant, nous n'avons pas observé de parents prendre l'initiative d'une conversation informelle avec les enseignantes pendant les 10 minutes avant ou après la classe. Quand les parents initient le contact avec l'enseignante, ils le font avant tout pour régler des questions pratiques ou d'organisation (voir Conus et Fahrni 2017). Les enseignantes nous font part de leur frustration face à la retenue des parents lors de ces moments de transition, comme par exemple cette enseignante du petit bâtiment:

E : Les parents sont très souvent euh là, dehors, mais très peu de parents s'inquiètent de comment leur enfant a passé la journée: "est-ce que ça a bien été, est-ce que, ah moi je l'ai trouvé par exemple fatigué, est-ce que vous avez remarqué quelque chose, oh moi je le trouve un petit peu plus pénible ou bien non je trouve justement qu'il est beaucoup plus ouvert, il parle maintenant il y a des mots en français, il aime, il aime mieux dessiner, mieux découper». Je trouve qu'il y a très très peu de retours sur ce, cet aspect de l'école.

C: Je suis pas sûre d'avoir compris, plutôt de votre côté, vous avez peu l'occasion de le dire aux parents, ou les parents viennent peu?

E : Les, les parents!

C: D'accord.

E: Les parents viennent très ra-, c'est souvent moi qui vais leur dire et ben tiens, aujourd'hui ben je trouve votre enfant il a fait un effort, parce que voilà, il a bien dessiné, il a bien découpé, mais c'est plus moi que les parents viennent chercher les informations. Moi je pense que pour les parents, tant que l'enfant est heureux, il a du plaisir, pour eux je pense que c'est, c'est presque suffisant.

(entretien enseignante $D, 04.11 .2013$ )

Dans leur interprétation du comportement des parents, les enseignantes montrent ainsi peu de sensibilité aux facteurs situationnels qui pourtant configurent fortement ces moments de transition et s'accordent mal avec l'objectif de construire une relation entre enseignantes et parents. Sans se rendre compte qu'elles ne sont elles-mêmes que très peu disponibles à l'échange avec les parents, accaparées par leurs tâches de contrôle, les enseignantes privilégient une attribution interne négative de la retenue des parents et l'expliquent par leur faible intérêt ou par des attentes limitées voire naïves envers la scolarité de leur enfant. Ce faisant, les enseignantes reprennent à leur compte le mythe de la démission parentale, souvent invoqué par les enseignants et pourtant déconstruit par de nombreux chercheurs (notamment Lightfoot 1978; Monceau 2014; Périer 2005). La complainte enseignante au sujet de la démission des parents relève d'une vision déficitaire des parents - en particulier ceux venant de groupes minoritaires - qui est constatée auprès d'enseignants par de nombreuses recherches (par exemple 
Boulanger, Larose, et Couturier 2010; Matthiesen 2016; Roy et Roxas 2011). Comme le prédit la théorie de l'accommodation de la communication (Gallois, Ogay, et Giles 2005) qui reprend ici les théories de l'attribution (Heider 1958; Kelly 1973), l'attribution interne négative du comportement de communication jugé inadéquat a pour conséquence une baisse de motivation à interagir à l'avenir avec la personne en particulier, et avec le groupe social auquel on l'identifie. Ainsi, le manque de reconnaissance par les enseignantes des facteurs situationnels qui limitent l'initiative des parents dans les contacts se traduit-il par une démotivation des enseignantes qui peuvent alors être tentées de sortir le moins possible de leur classe afin d'éviter ces situations qui ne répondent pas à leurs attentes. Les parents tendant également à se tenir en retrait en raison des contraintes situationnelles qui ne font qu'amplifier les difficultés qu'ils peuvent par ailleurs éprouver (notamment l'insécurité sur leurs compétences linguistiques, la peur de déranger ou un sentiment de manque de légitimité, voir Conus et Fahrni 2017), le malaise n'est jamais exprimé et il n'y a pas de métacommunication sur les difficultés rencontrées. L'ambiguïté s'accroît, l'incompréhension s'installe et chacun s'isole avec ses frustrations par rapport à la rencontre souhaitée, mais qui ne se fait pas. Pour autant, et peut-être qu'ici la variable culturelle du contexte helvétique joue son rôle, tout le monde prend garde à éviter le conflit en maintenant les apparences d'une bonne entente (Ogay 2017b). II est dès lors difficile de construire les bases d'une relation école-familles assez solide pour pouvoir négocier les inévitables tensions qui apparaîtront à un moment ou un autre de la scolarité.

\section{Conclusion}

A la lisière de la famille et de l'école, les moments quotidiens d'arrivée et de départ de l'école se révèlent bien moins anodins qu'on pourrait le penser a priori. Nos analyses de ces rapides moments de transition montrent la complexité de cet espace/temps ainsi que les conséquences délétères pour la relation écolefamille que peut avoir la non prise en compte de cette complexité. Dans ce moment très particulier où l'action des enseignantes se donne à voir aux parents et aussi aux collègues, aux chercheurs voire même aux passants, se nouent des enjeux de mise en scène et de quête de reconnaissance comme bon enseignant ou bon parent. La complexité de ce moment de l'entre-deux n'étant pas reconnue, le face-àface ne devient pas rencontre, mais échoue en dos à dos, engendrant son lot de frustrations qui configurent les relations à venir.

Pour commencer, il serait souhaitable que les professionnels responsables de l'aménagement de l'espace construit dans lequel se passe l'accueil et la sortie des élèves prennent conscience de l'importance d'offrir à la transition un espace favorable: lorsqu'il est trop restreint et s'apparente à un non-lieu (Augé 2010), l'espace est un obstacle significatif à la réalisation de deux objectifs que les acteurs tentent de concilier: le contrôle des enfants/élèves dans ce moment de transition et la construction d'une relation permettant la collaboration école-familles. Mais au-delà des contraintes posées par l'espace-construit, il y a l'interprétation que les acteurs font de ce moment et les actions qu'ils et elles entreprennent pour le faciliter, ou pas. L'ambiguïté inhérente à la situation de transition, où les rôles (notamment la tension entre 'relationner' et contrôler analysée ici) et les identités sont incertaines, demande à être reconnue et traitée, en particulier par les enseignants qui reçoivent les parents sur leur propre territoire et se trouvent ainsi dans la situation d'hôte. Ceci demanderait que l'institution scolaire valorise réellement l'action des enseignants en faveur de la collaboration école-familles, qui ne se décrète pas dans un article de loi mais demande un véritable investissement, au quotidien. La formation 
des enseignants, initiale comme continue, est bien sûr aussi concernée et devrait faire une plus large place aux aspects relationnels et communicationnels, qui ont également leur importance pour la qualité des apprentissages au cours de la scolarité.

\section{Références bibliographiques}

Augé, Marc. 2010. “Retour sur les « non-lieux." Communications 87 (1): 171-78. doi:10.3406/comm.2010.2631.

Blumer, Herbert. 1969. Symbolic Interactionism: Perspective and Method. Berkley: University of California Press.

Boulanger, Dany, François Larose, et Yves Couturier. 2010. "La logique déficitaire en intervention sociale auprès des parents : les pratiques professionnelles et les représentations sociales." Nouvelles pratiques sociales 23 (1): 152-76. doi:10.7202/1003174ar.

Bronfenbrenner, Urie. 1986. "Ecology of the family as a context for human development: Research perspectives". Developmental Psychology 22:723-42.

Conus, Xavier, et Laurent Fahrni. 2017. "Routine Communication between Teachers and Parents from Minority Groups: An Endless Misunderstanding?" Educational Review, novembre, 1-23. https://doi.org/10.1080/00131911.2017.1387098.

Conus, Xavier, et Tania Ogay. soumis et révisé. "Communication entre enseignants et parents minoritaires lors du premier entretien: effet-miroir d'extension et de restriction de rôles". In Regards critiques sur la relation école-famille(s), édité par Liliane Pelletier et Annick Lenoir.

Curchod-Ruedi, Denise, et Christiane Chessex-Viguet. 2012. "De la famille au cycle initial de la scolarité. Rupture ou transition ?" In Les transitions à l'école, édité par Pierre Curchod, Pierre-André Doudin, et Louise Lafortune, 12-31. Québec: Presses de l'Université du Québec.

Da Cunha, Antonio, et Sandra Guinand. 2014. Qualité urbaine, justice spatiale et projet: Ménager la ville. Lausanne : PPUR.

Dartiguenave, Jean-Yves. 2012. “Rituel et liminarité". Sociétés, nº 115 (juin):81-93. https://doi.org/10.3917/soc.115.0081.

Deschamps, Jean-Claude, et Jean-Léon Beauvois. 1996. Des attitudes aux attributions. Sur la construction de la réalité sociale. Vol. II. La psychologie sociale. Grenoble: Presses universitaires de Grenoble.

État de Fribourg. 1985. Loi du 23 mai 1985 sur la scolarité obligatoire (loi scolaire, LS). http://www.lexfind.ch/dtah/85068/3/411.0.1.pdf.

- - . 1986. Règlement d'exécution de la loi scolaire (RLS). 411.0.11. https://edudoc.ch/record/136/files/FR.pdf.

- - _. 2014. Loi du 9 septembre 2014 sur la scolarité obligatoire (loi scolaire, LS). http://bdlf.fr.ch/frontend/versions/4312.

- - . 2016. Règlement de la loi sur la scolarité obligatoire (RLS). https://bdlf.fr.ch/frontend/versions/4734?locale=fr.

Frame, Alexander. 2013. Communication et interculturalité : cultures et interactions interpersonnelles. Paris: Lavoisier.

Gallois, Cynthia, Tania Ogay, et Howard Giles. 2005. "Communication Accommodation Theory: a look back and a look ahead". In Theorizing about intercultural communication, édité par W. B. Gudykunst, 121-48. Thousand Oaks: Sage.

Goffman, Erwing. 1973. La mise en scène de la vie quotidienne. La présentation de soi. Paris: Editions de Minuit. 
Gudykunst, William B. 2005. “An Anxiety/Uncertainty Management (AUM) theory of effective communication. Making the mesh of the net finer". In Theorizing about intercultural communication, édité par William B. Gudykunst, 281-322. Thousand Oaks: Sage.

Hampden-Thompson, Gillian, et Claudia Galindo. 2017. "School-family Relationships, School Satisfaction and the Academic Achievement of Young People". Educational Review 69 (2):248-65. https://doi.org/10.1080/00131911.2016.1207613.

Heider, Fritz. 1958. The psychology of interpersonal relations. New York: John Wiley.

Kelly, Harold H. 1973. "The process of causal attribution". American Psychologist 28: 107-28.

Larivée, Serge. J., Jean-Claude Kalubi, et Bernard Terrisse. 2006. "La collaboration école-famille en contexte d'inclusion: entre obstacles, risques et facteurs de réussite". Revue des sciences de l'éducation 32: 525-43.

Lightfoot, Sarah Lawrence. 1978. Worlds Apart: Relationships between Families and Schools. New York: Basic.

Marcel, Jean-François. 2002. "Approches ethnographiques des pratiques enseignantes durant les temps interstitiels". Revue de recherches en éducation 30: 103-21.

Matthiesen, Noomi Christine Linde. 2016. "Working together in a deficit logic: home-school partnerships with Somali diaspora parents". Race Ethnicity and Education 20 (4): 495-507. doi:10.1080/13613324.2015.1134469.

Mead, George Herbert. 1934. Mind, self and society. Chicago: University of Chicago Press.

Miles, Matthew, et Michael A. Huberman. 2003. Analyse des données qualitatives. 2e éd. Bruxelles: De Boeck Université.

Monceau, Gilles. 2014. "Effets imprévus des dispositifs visant à rapprocher les parents éloignés de l'École". Education et sociétés 34 (2): 71-85. doi:10.3917/es.034.0071.

Nunez Moscoso, Javier, et Tania Ogay. 2016. "L'entretien enseignant-famille à l'école enfantine : quelles dynamiques organisationnelles, quels savoirs mobilisés?" Revue des sciences de l'éducation 42 (1): 147-81.

Ogay, Tania. 2017a. "Quand l'enfant devient élève, et les parents, parents d'élèves. Construction de la relation entre les familles et l'école lors de l'entrée à l'école (recherche COREL)". Rapport final à I'intention du Fonds National Suisse de la Recherche. Fribourg: Université de Fribourg, Département des Sciences de l'éducation. http://www.unifr.ch/ipg/assets/files/DocSite/COREL_rapportFNS.pdf

- - . 2017b. "L'entrée à l'école, berceau de l'alliance éducative entre l'école et les familles? Le rôle perturbateur des implicites de l'école". Revue suisse des Sciences de l'éducation 39 (2): 336-51.

Paillé, Pierre, et Alex Mucchielli. 2012. L'analyse qualitative en sciences humaines et sociales. Paris: A. Colin.

Patrikakou, Evanthia N, Roger P. Weissberg, Sam Redding, et Herbert J. Walberg, éd. 2005. School-Family Partnerships for Children's Success. New York: Teachers College Press.

Payet, Jean-Paul. 2016. "Postface. La singularité ethnographique". In Ethnographie de l'école. Les coulisses des institutions scolaires et socio-éducatives, édité par Jean-Paul Payet, 203-20. Rennes: Presses universitaires de Rennes.

Périer, Pierre. 2005. Ecole et familles populaires: sociologie d'un différend. Rennes: Presses universitaires de Rennes.

- - . 2015. "L'enfant entre deux mondes : disqualification parentale et autonomisation scolaire." Les Sciences de l'éducation - Pour l'Ėre nouvelle 48:105-26. https://doi.org/10.3917/lsdle.481.0105.

- - . 2017. "Les familles immigrées aux marges de l'école. Dépendance et mobilisation des parents dans le contexte d'un quartier populaire." Cahiers de la recherche sur l'éducation et les savoirs 16:229-51. 
Rimm-Kaufman, Sara E., et Robert C. Pianta. 2000. "An ecological perspective on the transition to kindergarten : a theoretical framework to guide empirical research". Journal of Applied developmental psychology 21: 491-511.

Roy, Laura, et Kevin Roxas. 2011. "Whose deficit is this anyhow? Exploring counter-stories of Somali Bantu refugees' experiences in 'doing school' ". Harvard Educational Review 81 (3): 521-42.

Scalambrin, Laure, et Tania Ogay. 2014. " 'Votre enfant dans ma classe'. Quel partenariat parentsenseignante à l'issue du premier entretien ?" Education et sociétés 34 (2): 23-38. doi:10.3917/es.034.0023.

Soulier, Nicolas. 2012. Reconquérir les rues: exemples à travers le monde et pistes d'actions: Pour des villes où l'on aimerait habiter. Paris: Ulmer.

Super, Charles, et Sara Harkness. 1986. "The developmental niche: a conceptualization at the interface of child and culture". International Journal of Behavioral Development 9(4):545-70.

Ting-Toomey, Stella. 2004. "Translating conflict face-negotiation theory into practice". In Handbook of intercultural training, édité par Dan Landis, Janet M. Bennett, et Milton J. Bennett, 217-48. Thousand Oaks, CA: Sage.

Turner, Victor W. 1990. Le phénomène rituel. Structure et contre-structure. Paris: PUF.

Van Gennep, Arnold. 1909. Les rites de passage: étude systématique des rites. Paris: E. Nourry.

Watzlawick, Paul, Janet H. Beavin, et Don D. Jackson. 1972. Une logique de la communication. Paris: Seuil.

Zittoun, Tania. 2012. "Une psychologie des transitions: des ruptures aux ressources." In Les transitions à l'école, édité par Pierre Curchod, Pierre-André Doudin, et Louise Lafortune, 263-79. Québec:

Presses de l'Université du Québec. 